This item was submitted to Loughborough's Research Repository by the author.

Items in Figshare are protected by copyright, with all rights reserved, unless otherwise indicated.

The implementation of an AR (augmented reality) approach to support mammographic interpretation training - an initial feasibility study

PLEASE CITE THE PUBLISHED VERSION

http://dx.doi.org/10.1117/12.2255833

PUBLISHER

(c) SPIE

VERSION

VoR (Version of Record)

LICENCE

CC BY-NC-ND 4.0

REPOSITORY RECORD

Tang, Qiang, Yan Chen, and Alastair G. Gale. 2019. "The Implementation of an AR (augmented Reality) Approach to Support Mammographic Interpretation Training - an Initial Feasibility Study". figshare. https://hdl.handle.net/2134/24636. 


\title{
The implementation of an AR (augmented reality) approach to support mammographic interpretation training - an initial feasibility study
}

\author{
Qiang Tang ${ }^{\mathrm{a}}$, Yan Chen ${ }^{*}$, and Alastair G Gale ${ }^{\mathrm{a}}$ \\ ${ }^{a}$ Applied Vision Research Centre, Loughborough University, Loughborough, UK
}

\begin{abstract}
Appropriate feedback plays an important role in optimising mammographic interpretation training whilst also ensuring good interpretation performance. The traditional keyboard, mouse and workstation technical approach has a critical limitation in providing supplementary image-related information and providing complex feedback in real time. Augmented Reality (AR) provides a possible superior approach in this situation, as feedback can be provided directly overlaying the displayed mammographic images so making a generic approach which can also be vendor neutral. In this study, radiological feedback was dynamically remapped virtually into the real world, using perspective transformation, in order to provide a richer user experience in mammographic interpretation training. This is an initial attempt of an AR approach to dynamically superimpose pre-defined feedback information of a DICOM image on top of a radiologist's view, whilst the radiologist is examining images on a clinical workstation. The study demonstrates the feasibility of the approach, although there are limitations on interactive operations which are due to the hardware used. The results of this fully functional approach provide appropriate feedback/image correspondence in a simulated mammographic interpretation environment. Thus, it is argued that employing AR is a feasible way to provide rich feedback in the delivery of mammographic interpretation training.
\end{abstract}

Keywords: AR, Augmented Reality, mammographic interpretation, training

\section{INTRODUCTION}

Various previous research ${ }^{1,2}$ suggests that appropriate Human-Computer Interaction (HCI) could bring a considerable improvement in medical imaging interpretation training. An example is online training, which is becoming more available, with real-time expert supervision or guidance. Consequently, intuitive training with real-time user interaction and feedback could be a very effective approach. The current work develops a device-independent AR approach which can provide mammographic image interpretation training with rich feedback.

The motivation for this particular work is the perceived need to incorporate both enhanced image and text feedback information, together with the original radiological image, in a suitable format that enhances user interaction. The UK's breast screening self-assessment scheme (Personal Performance in Mammographic Screening - PERFORMS ${ }^{\circledR}$ ) clearly shows that training plays an important role in maintaining skill levels of mammographic interpretation. ${ }^{3}$ Appropriate mammographic training is a key practical challenge due to the relative shortage of availability of clinical workstations for training purposes ${ }^{4}$ and realistic screening experience could be a key aspect of successful training. ${ }^{5}$

In the PERFORMS ${ }^{\circledR}$ scheme full field digital mammograms (FFDM) are examined on suitable high resolution mammographic monitors and users make decisions on each mammographic case using an interactive web-based system which can run either on the monitor or on any alternative computing device. We set out to determine whether the user interaction with the cases in this scheme could be wholly incorporated within an AR approach and the development is described here. If successful then further training approaches in radiology using this AR system could be easily developed.

\footnotetext{
*Y.Chen@lboro.ac.uk
}

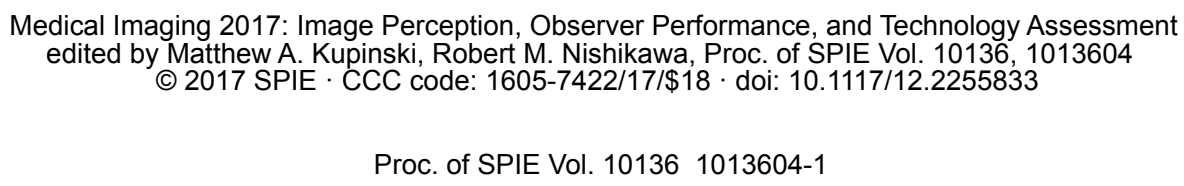




\section{METHOD}

There are various steps in constructing a suitable AR system and the conceptual stages of the initial AR structure which was developed are shown in Figure 1 and are described below in numbered steps:-

Firstly, a series of mammographic cases are displayed one at a time on the workstation and examined by the radiologist (1). Each mammographic DICOM file may be normal or contain malignant or benign radiographic features. If either of the latter then the case will have known feedback marks identifying areas of interest associated with the case. These marks would have been first provided by expert radiologists or by the experimenter, based on known information such as the case pathology data. Such feedback information is normally not visible to the radiologist when examining the images on the workstation.

In the current approach the radiologist views the mammographic images whilst wearing the Google Glass AR system (2) which then enables appropriate feedback information to be displayed on the AR system. This presupposes that the case being examined is appropriately identified to the AR system. Any AR approach comprises a head mounted forward facing camera to capture the scene being viewed by the observer coupled with a display system which permits the feedback information to be appropriately shown overlaying the viewed scene. However, in order for any image-related feedback information to be displayed via the AR system then this information has to be appropriately superimposed on the workstation mammographic images. Such imposition has to allow for the radiologist to move with respect to the workstation both laterally, as well as anterior/posterior movements, whilst also allowing for torsional head movements.

This is achieved by the following approach. Image processing (object recognition) of the AR camera image is implemented in order to detect the actual screen area of the workstation, which naturally moves as the radiologist moves their head (3). The screen contents are then extracted into time-sequenced images; these images are captured in real time so that a transformation has to be applied for each one. Perspective transformations (3) are then applied to deduce planar images and then make the feedback marks visible to the radiologist via the AR device. Figure 2 shows the procedure of using perspective transformation to transform an irregular image (left) to a regular shape image (right). In the middle of the figure, the $[u, v]$ coordinate system stands for a $2 \mathrm{D}$ matrix which is $[\mathrm{u}, \mathrm{v}, \mathrm{w}]$ in $3 \mathrm{D}$ space. The perspective transformation matrix is a general $3 * 3$ matrix expression for effective spatial transformations (including scaling, shearing, rotation, reflection, translation and perspective in space). ${ }^{6}$ An inverse transformation of the above is then applied (4).

Therefore the final results match with the perspective view in the real world. The marks are calculated and registered with the transformed images (step 4.1 in Figure 1). The results are then presented via AR to the radiologist (5). All such vision changes would happen on the Google Glass screen and are only visible to the radiologist wearing the $\mathrm{AR}$ device.

The initial approach was set up in one of our research laboratories using a GE mammographic workstation, and studies were initialised with Google Glass, as an exemplar AR system, and image processing completed using an Android virtual machine. Unfortunately, Google Glass has no capability for any complex image processing. However, to maximally simulate a real mammographic reporting scenario, Google Glass was used to capture images from the radiologist's viewpoint. Ideally, a pair of AR glasses (or HMD Head Mounted Display) would be responsible for both capturing real-time images and to perform all image processing. A final functional AR system such as the Microsoft Hololens is a probable end target device.

\subsection{Accuracy}

For any AR system to be useful then any displayed information, particularly overlaying image-related information (such as the boundary of an abnormality) must be appropriately and accurately co-registered with the mammographic images as displayed on the workstation. The potential accuracy of this method is limited by two metrics:

- AR camera resolution

- AR display resolution 


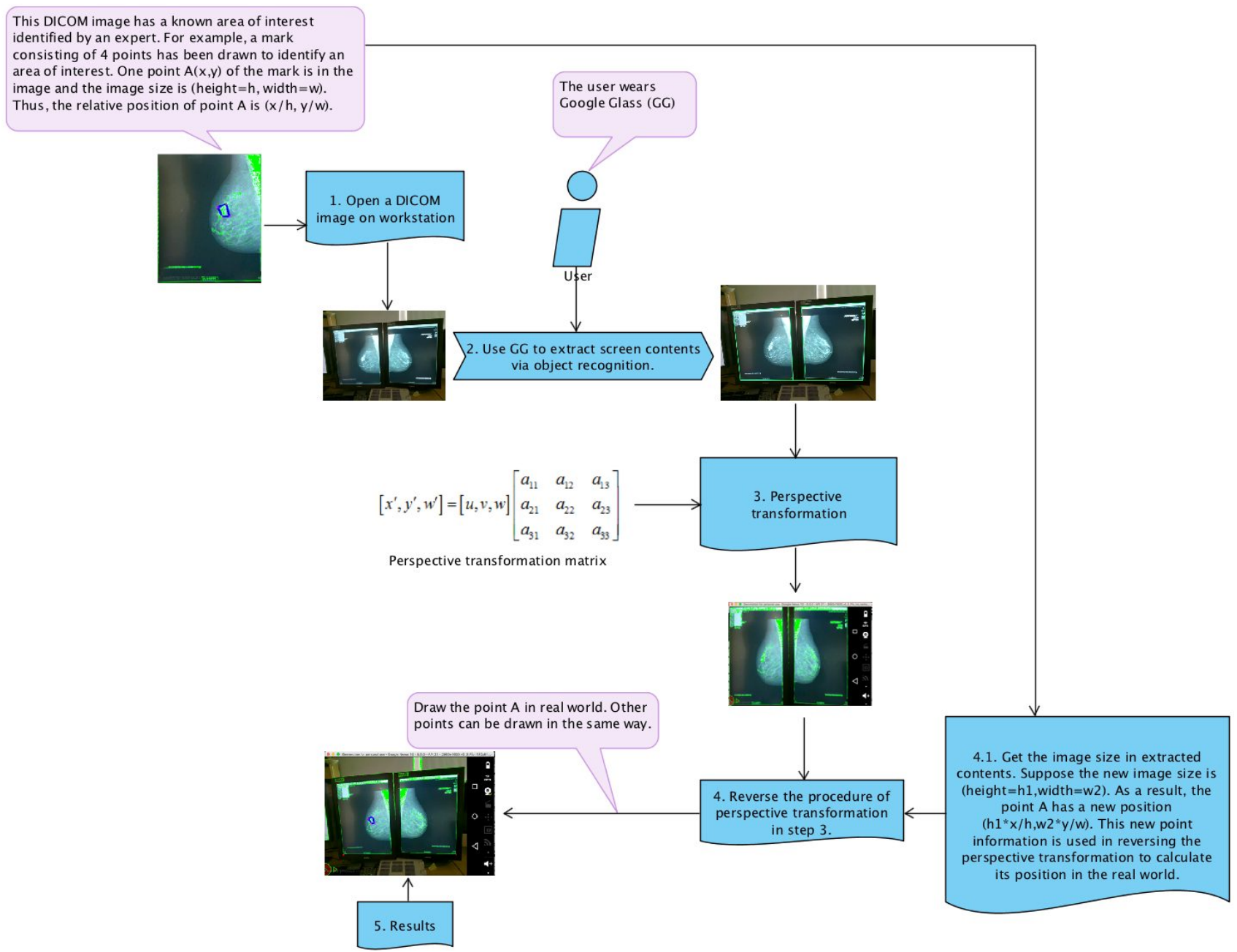

Figure 1. The implementation process with numbered steps.

Each measure consists of both a horizontal and a vertical measure which increases in proportion to the number of pixels of the workstation screen area as captured by the camera. The camera and display resolution of Google Glass are constants as follows:

- Google Glass camera: $720 p(1280 * 720 p x)$;

- Google Glass display: $640 * 360 p x$.

The mammographic screening environment is a dark room so that the AR camera images have high contrast and low noise. This leads to suitable detection using the Hough transform which is a proven and robust algorithm for finding lines even in noisy images. ${ }^{7,8}$ As a result, the experimental accuracy of the hardware is gauged to be at a pixel level (in our experiments, the accuracy is 1 pixel, digital images are aliasing graphics, and this could be improved by sub-pixel compensation).

\subsection{Improvements}

Early trials of the approach described above demonstrated that accurate screen detection was possible, therefore enabling superimposition of feedback marks via AR, however a Google Glass like device did not have the computing power necessary for a workable AR system. 


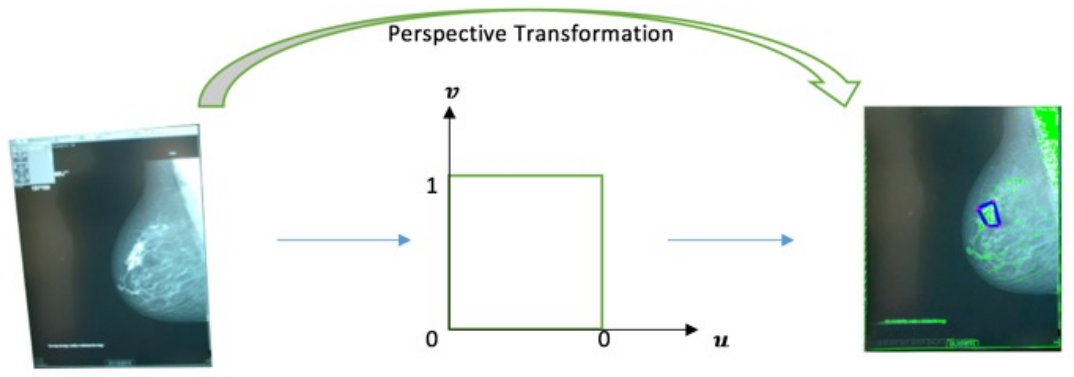

Screen contents on workstation (in first-person or third-person view)

Screen of Google Glass

Figure 2. The procedure of perspective transformation in a $2 \mathrm{D}$ space. ${ }^{6}$

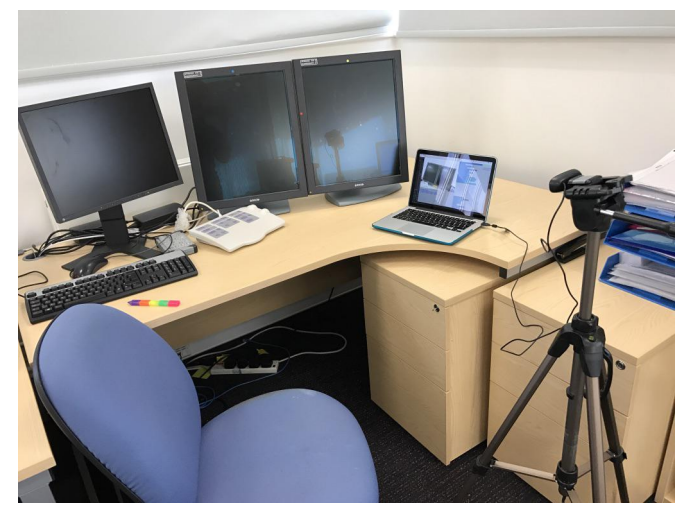

Figure 3. A laptop-based AR environment contains: a laptop, an external camera and a mammographic workstation.

Consequently, an alternative approach to the AR development was taken by substituting a conventional laptop and an external camera to build a simulated AR environment (Figure 3). The camera functions as the head mounted AR camera and the laptop monitor as the overlay AR screen. This approach allows user behaviour to be tracked and analysed in real time. At the same time, such a simulated AR environment still allows unobstructed user interaction with the mammographic workstation. The only difference from a wearable AR device is that such a simulated AR environment cannot present augmented views directly to the user who can only see such results on the laptop screen. To allow interactions and feedback, a further OCR approach and a natural writing method are added to the laptop-based AR. The approach is described below.

As before, firstly an image processing procedure identifies and extracts the screen area of the mammographic workstation. OCR is then used to identify the mammographic case number displayed on the workstation images which then enables synchronisation between the workstation and the laptop so that the same mammographic case loaded on the workstation is accessed from the PERFORMS ${ }^{\circledR}$ database and displayed on the laptop. If the case is abnormal then the laptop image will have associated with it radiological and pathological findings together with demarcated abnormality outline co-ordinates. By then performing a perspective transformation (as in Figure 2) between the workstation screen area and the loaded laptop mammographic images, any positional finding or pathology can be plotted on the laptop at its appropriate position overlaying the mammographic case. Figure 4, taken from the fixed camera position, shows an example of this. Having identified the screen areas then OCR identifies which case is being viewed (currently this takes four seconds) which here has an ID of CXR,01. Then the associated clinical information for this case is plotted at an appropriate position. On the right of figure 4 is shown the distribution information of target monitors which are measured by their screen edges.

In this set up some form of suitable interaction is required which allows the radiologist to identify areas on the workstation and communicate these areas to the AR system. Consequently, the system is designed to recognise 
a stylus held by the radiologist and log its location on the workstation images (Figure 5). To do this the stylus is placed in front of the camera and the stylus tip, its button and the stylus tail are all recognised in turn by identifying them to the system by appropriate mouse clicking. Quickly moving the stylus towards or away from a workstation monitor will either make the stylus available or unavailable. If the stylus tip is orientated to the left of the workstation then the radiologist can use the stylus to annotate the case as in normal writing. To alter any annotations then these can be erased by having the stylus oriented with the stylus tail pointing to the left. The stylus can be further implemented with any other desired functions.

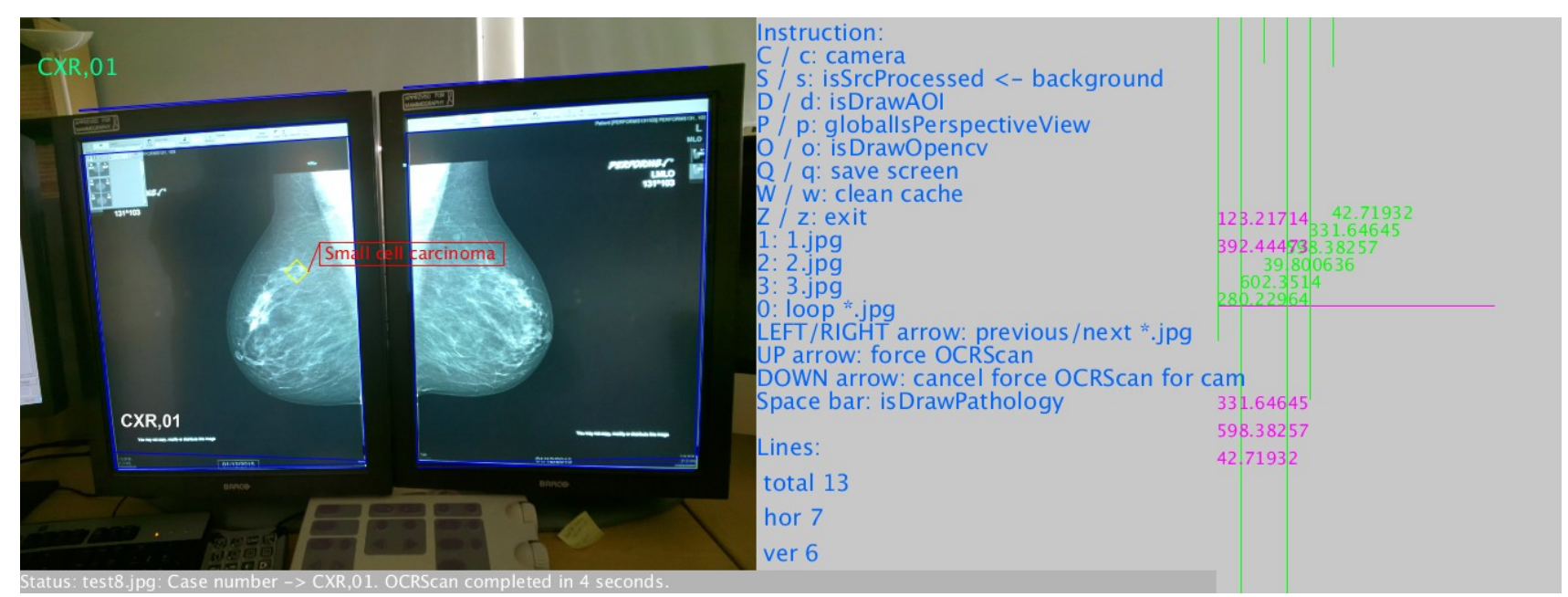

Figure 4. Augmented view.

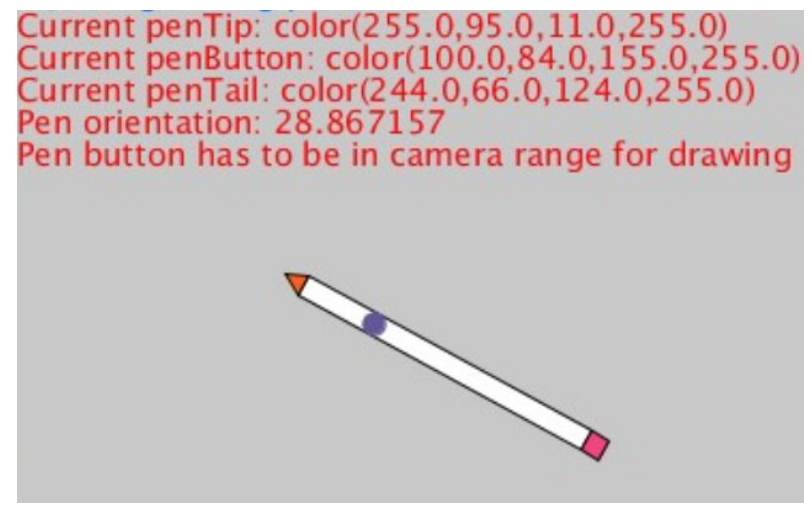

Figure 5. A registered stylus in the system with an orange tip, purple button and pink tail. The orientation of the tip indicates a drawing status if it appears within a workstation screen area.

\subsection{Interaction accuracy}

Interactions between a machine and a person are generally affected by the accuracy of detection and the stability of hand operation. To test the accuracy of user input (by stylus) through the laptop-based AR, two types of studies were designed:

a) Visual cues guided user input (Figure 6): in this case, different coloured crosses were used to represent the positions of a stylus's components. While a user is moving the stylus, the positions and orientations of it can be viewed via the laptop. Here the user has to pay attention to both the monitor of the mammographic workstation as well as the laptop. This approach works in a similar method to a conventional mouse. The stability of hand-held input devices will significantly affect the accuracy of user input. 
b) Non-visual cues guided user input: in this case, the user only pays attention to the mammographic workstation monitors. With a laptop-based AR approach, the user does not know the final results of any interaction in real time and so cannot review any interaction results immediately. Nevertheless, user input can be recorded for later review.

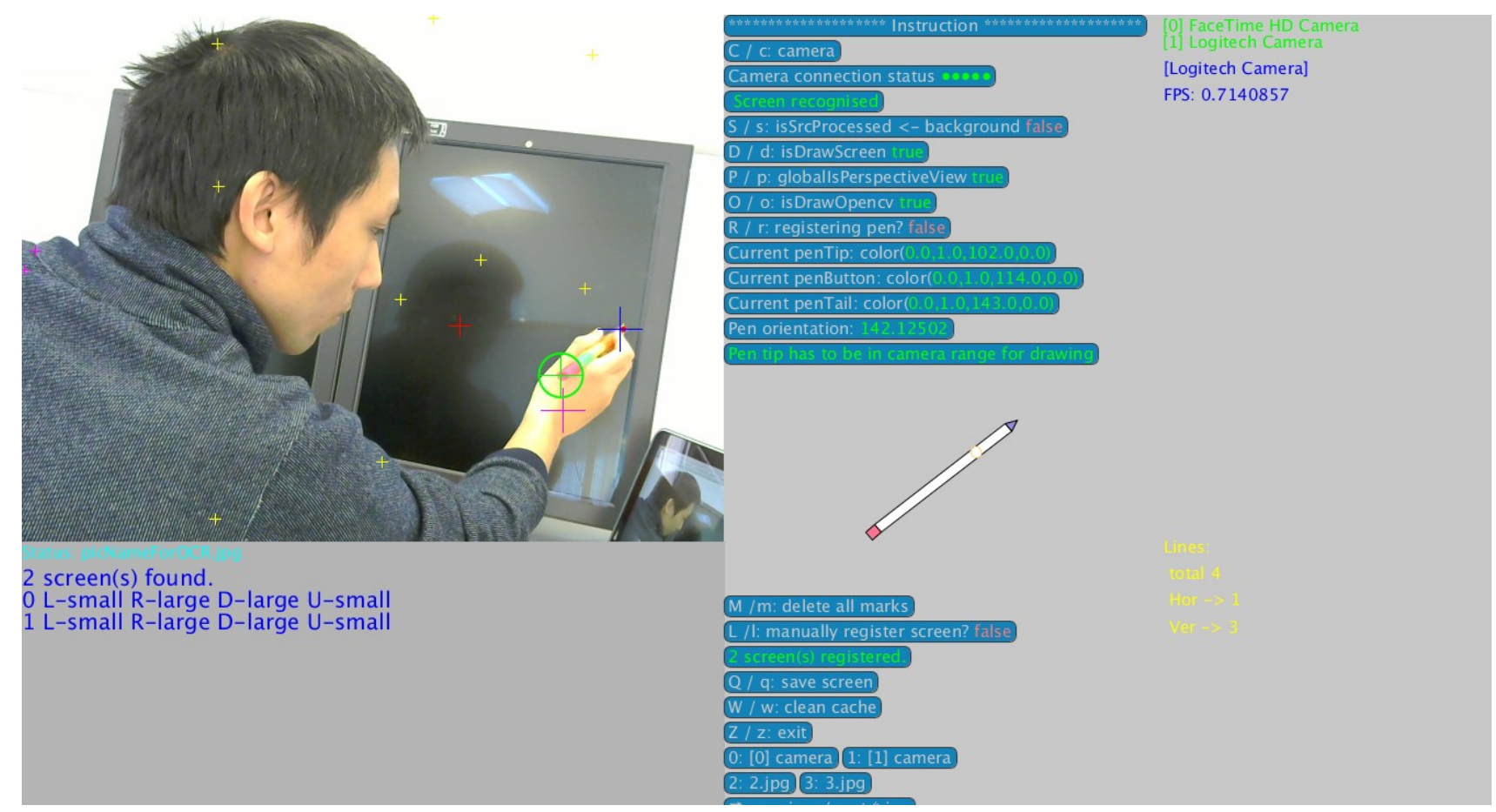

Figure 6. A participant identifying points that have been randomly computer generated. The registered stylus has a blue cross indicating its tip position, a pink cross indicating its button position (in this case, the button function is disabled) and a green cross indicating its eraser position.

\subsubsection{Results}

Initial results show that the visual cues guided approach has a higher accuracy than the non-visual cues guided approach. The stylus position tracking system implemented on the laptop-based AR application showed an average accuracy of \pm 5 pixels while a user keeps hovering the tip of a stylus above a randomly generated point on the workstation monitors. Furthermore, results can be improved by utilising a high resolution camera and large monitor which can provide detailed images can be obviously conducive to a more accurate result through comparing results with different sized monitors and cameras with different resolutions. The results are consistent with $^{9}$ which transcribed feature positions on a mammographic image to a simplified copy.

\subsection{Limitations}

Currently, the approach does not allow for zooming or panning of the mammographic images on the workstation. Moreover, AR approaches require complicated image processing procedures so that high performance hardware is required. The configuration used in our work to date is a laptop with $2.7 \mathrm{GHz}$ Intel Core i5, 8GB memory and an integrated GPU. Such a laptop does not have capability for graphic acceleration so that image processing currently has a high time cost.

\section{RESULTS}

This work proposes an initial approach to AR-based mammographic interpretation training. AR in medical imaging is very much still in its infancy, although in surgery AR has been shown to be very useful for displaying 
extra information. ${ }^{10}$ The present work presents a novel way of using AR to deliver mammographic interpretation training. Also, it provides rich user interaction and feedback as needed in screening. Google Glass was first used as an AR device to visualise feedback annotations overlaid on workstation mammographic case. The research development enabled synchronising of the mammographic images displayed on both a workstation screen and an AR device, although zooming and panning of the workstation images are currently not mirrored on the AR system. A stylus was enabled as an input device to enable an observer to annotate workstation images. Initially a dark room was required to enable workstation screen detection, although subsequently this low ambient lighting requirement was not needed. A series of radiologist studies is currently underway which utilise this AR approach to examine further AR aspects useful to the breast radiologist. Though it is feasible to integrate real time eye movement recording with manipulation in medical imaging practice, our work to date has simply simulated possible manipulations with varied head movements but not changing eye gaze direction. The reason for this is because Google Glass does not have an integrated eye tracker. This is one particular aspect which we are currently developing.

\section{DISCUSSION}

Augmented Reality has the ability to deliver situated learning opportunities and also to facilitate the perception of complex situations in medical education. ${ }^{11}$ However, an AR application must also be able to provide appropriate feedback to a user. ${ }^{12}$ Due to hardware computing capability and cost, wearable AR devices will probably be slow to be widely adopted in breast screening. In the current exemplar case, a compromise of laptop-based AR has been used for the development of mammographic interpretation training to overcome Google Glass limitations. However, a high performance laptop is still required because of the calculations required for mammographic image processing.

By using adaptive threshold and computing the distributions of screen edges, it is possible to employ such an AR facility for different training scenes with nonspecific devices. For instance, a tablet and a conventional workstation can be jointly used and display different clinical information as desired. Nevertheless, interaction on a tablet is not as accurate as that on a large sized conventional workstation. Additionally, handwriting skills significantly affect the accuracy of manipulations while using an input device such as a stylus. ${ }^{13}$

\section{CONCLUSIONS}

This study demonstrates that it is feasible to deliver mammographic interpretation training with the support of AR. Future work will concentrate on manipulations using other AR devices. The prototype described here is fully functional yet the current development platform could not realise its full potential. Hardware is a key aspect in successfully adopting AR devices in medical imaging. A laptop-based AR has been used as an initial alternative, considering the current limited availability of a suitable wearable AR device.

\section{REFERENCES}

[1] Preim, B., "HCI in Medical Visualization," Dagstuhl Follow-Ups 2, 310 (2011).

[2] Weiss, D. L., Siddiqui, K. M., and Scopelliti, J., "Radiologist assessment of PACS user interface devices.," Journal of the American College of Radiology : JACR 3, 265-273 (Apr. 2006).

[3] Chen, Y., Gale, A., and Scott, H., "Mammographic interpretation training: what exactly do film-readers want?," Breast Cancer Research 10(Suppl 3), P85 (2008).

[4] Chen, Y. and Gale, A., "Intelligent Computing Applications Based on Eye Gaze: Their Role in Medical Image Interpretation," International Conference on Intelligent Computing 93(Chapter 43), 320-325 (2010).

[5] Gale, A. G., "PERFORMS: a self-assessment scheme for radiologists in breast screening," Seminars in Breast Disease 6(3), 148-152 (2003).

[6] Wolberg, G., "Geometric Transformation Techniques for Digital Images: a Survey," Department of Computer Science, Columbia University (1988).

[7] Ji, Q. and Haralick, R. M., "Error propagation for the Hough transform," Pattern Recognition Letters 22, 813-823 (May 2001). 
[8] Zeevi, A. and Goldenshluger, A., "The Hough transform estimator," The Annals of Statistics 32, 1908-1932 (Oct. 2004).

[9] Hatton, J. W., Wooding, D. S., Gale, A. G., and Purdy, K. J., "Accuracy of transcribing locations on mammograms: implications for the user interface of a system to record and assess breast screening decisions," in [Proceedings of the SPIE], Chakraborty, D. P. and Krupinski, E. A., eds., 32-41, Univ. of Derby, United Kingdom, SPIE (May 2003).

[10] Meola, A., Cutolo, F., Carbone, M., Cagnazzo, F., Ferrari, M., and Ferrari, V., "Augmented reality in neurosurgery: a systematic review.," Neurosurgical review , 1-12 (May 2016).

[11] Kamphuis, C., Barsom, E., Schijven, M., and Christoph, N., "Augmented reality in medical education?," Perspectives on Medical Education 3, 300-311 (Jan. 2014).

[12] Barsom, E. Z., Graafland, M., and Schijven, M. P., "Systematic review on the effectiveness of augmented reality applications in medical training," Surgical endoscopy 30, 4174-4183 (Feb. 2016).

[13] Cornhill, H. and Case-Smith, J., "Factors that relate to good and poor handwriting.," The American journal of occupational therapy : official publication of the American Occupational Therapy Association 50, 732-739 (Oct. 1996). 